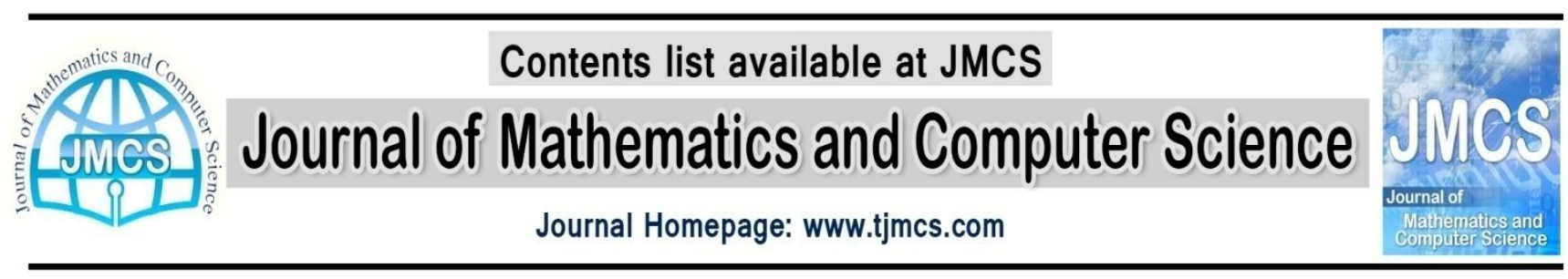

\title{
A meshless method for solving delay differential equation using radial basis functions with error analysis
}

\author{
F. Ghomanjani ${ }^{1}$, F. Akhavan Ghassabzade ${ }^{2}$ \\ ${ }^{1}$ Young Researchers and Elite Club, Mashhad Branch, Islamic Azad University, Mashhad, Iran. \\ ${ }^{2}$ Department of Applied Mathematics, Faculty of Mathematical Sciences, Ferdowsi \\ University of Mashhad, Mashhad, Iran.
}

Article history:

E-mail: fatemeghomanjani@gmail.com

Received April 2014

Accepted May 2014

Available online June 2014

\begin{abstract}
In this paper, we present a numerical method for solving delay differential equations (DDEs). The method utilizes radial basis functions (RBFs). Error analysis is presented for this method. Finally, numerical examples are included to show the validity and efficiency of the new technique for solving DDEs.

Keywords: Radial basis functions (RBFs), Delay differential equations, time delay systems.
\end{abstract}

\section{Introduction}

Delay differential equations are type of differential equations where the time derivatives at the current time depend on the solution, and possibly its derivatives, at previous times. A class of such equations, which involve derivatives with delays as well as the solution itself, has been called neutral DDEs over the past century (see $[1,2])$. Systems with time delay occur frequently in mechanical and electrical systems, population, industrial process, economic growth, neural networks, etc. Many researchers have tried various methods of optimizing linear time delay systems [3].

The basic theory concerning the stable factors and works on fundamental theory, e.g., existence and uniqueness of solutions, was presented in [1,2]. Since then, DDE have been extensively studied in recent decades and a great number of monographs have been published including significant works on dynamics of DDEs by Hale and Lunel [4], on stability by Niculescu [5], and so on. The interest in study of DDEs is caused by the fact that many processes have time-delays and have been models for better representations by systems of DDEs in science, engineering, economics, etc. Such systems, how-ever, are still not feasible to actively analyze and control precisely, thus, the study of systems of DDEs has actively been conducted over the recent decades (see $[1,2]$ ).

The orthogonal functions and polynomial series have been developed for solving various problems of dynamical systems such as system analysis, parameter identification, optimal control, etc [1, 6, 7]. The main idea of this technique is that it reduces these problems to those of solving a system of algebraic 
equations and thus it greatly simplifies the problem. The approach is based on converting the differential equations into an integral equation through integration. The state and/or control involved in the equation are approximated by finite terms of orthogonal series and by using the operational matrix of integration the integral operations are eliminated. The form of the operational matrix of integration depends on the particular choice of the orthogonal functions like Walsh functions [4], Block-pulse functions [8], Laguerre series [9], Jacobi series [10], Fourier series [11], Bessel series [12], Taylor series [13], Shifted Legendre [14], Chebyshev polynomials [15] and Hermite polynomials [16]. In this study, we use wavelet functions to approximate both the control and state functions. It avoids the difficult integral equations created from variational methods reducing the problem to the solution of an algebraic system of equations, thus providing a computationally more efficient approach. Wavelet functions possess useful properties such as orthogonality, compact support and exact representation of polynomials to a certain degree such as Haar [17], Legendre [18] and Sine-cosine [19] wavelets.

The outline of the paper is as follows. In Section 2, we review some basic formulations of RBFs. Numerical examples are given in Section 3. Finally, we conclude the article in Section 4.

\section{An outline of RBFs}

In this section we simply introduce some elementary knowledge about radial basis functions for more details see [20, 21, 22, 23]. In 1990, Kansa [20] introduced a novel approach for solving partial differential equations (PDE) by collocation motivated by advances in function approximation using radial basis function (RBFs). In this approach, the solution is approximated using RBFs as a trial functions and collocation technique is used to compute the undetermined coefficients. In the following radial basis functions are defined in any number of dimensions [24, 25].

Definition 1. A function $\phi: R^{d} \rightarrow R$ is called radial basis provided there exists a univariate function $\psi:[0, \infty) \rightarrow R$ such that $\phi(x)=\psi(r)$ where $r=\|x\|$ and $\|$.$\| is some norm on R^{d}$, usually the Euclidean norm. Some well-known RBFs are listed in Table 1.

Table 1: Some well-known radial basis functions.

\begin{tabular}{|lc|}
\hline Name of RBF & Definition \\
\hline Multiquadratics (MQ) & $\phi(r)=\sqrt{r^{2}+c^{2}}$ \\
Inverse multiquadratics (IMQ) & $\phi(r)=1 /\left(\sqrt{r^{2}+c^{2}}\right)$ \\
Thin plate splines(TPS) & $\phi(r)=(-1)^{k+1} r^{2 k} \log (r), k \in N$ \\
Gaussians (GA) & $\phi(r)=-\exp (-c r)$ \\
Inverse quartics (IQ) & $\phi(r)=1 /\left(r^{2}+c^{2}\right)$ \\
\hline
\end{tabular}

\subsection{Interpolation by RBFs}

The process of interpolation using a radial basis function is as follow.

Let $\left\{x_{1}, x_{2}, \ldots, x_{N}\right\}$ be a given set of distinct nodal points in $D \subset R^{d}$. The approximation of a function $f(x)$, using radial basis function $\psi(r)=\phi(|| x||)$, may be written as a linear combination of the form $[24,25]:$

$$
(S f)(x)=\sum_{x_{i} \in X} \lambda_{i} \phi\left(|| x-x_{i}||\right)+p(x), \quad x \in R^{d},
$$

for $S f$ to interpolate $f$ the real number $\lambda_{i}$ and polynomial $p$ must be chosen to satisfy the system

$$
\left\{\begin{array}{cc}
(S f)\left(x_{i}\right)=f\left(x_{i}\right), & x_{i} \in X, \\
\sum_{x_{i} \in X} \lambda_{i} q\left(x_{i}\right)=0, & \text { for all } q \in \pi_{m-1}^{d},
\end{array}\right.
$$

where $\pi_{m-1}^{d}$ denotes all polynomial on $R^{d}$ of total degree at most $m-1$ (see [26]). 


\section{Numerical Examples}

In this section, we give some computational results of numerical experiments with methods based on the preceding section.

Example 1. As a practical example, we consider Evens and Raslan [27] the following pantograph delay equation:

$$
\begin{gathered}
u^{\prime}(t)=\frac{1}{2} \exp \left(\frac{t}{2}\right) u\left(\frac{t}{2}\right)+\frac{1}{2} u(t), 0 \leq t \leq 1, \\
u(0)=1,
\end{gathered}
$$

where the exact solution of above equation is $u(t)=\exp (t)$ (see [28]). With the presented method, the graph of error is plotted in Fig. 1.

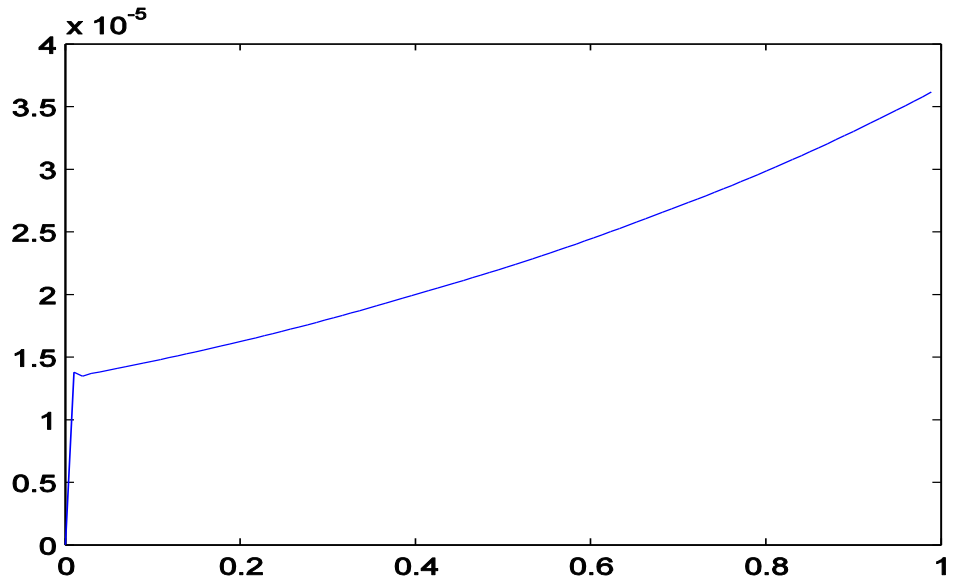

Figure 1: The graph error for Example 1

Example 2. Consider the following second order linear DDE

$$
\begin{gathered}
u^{\prime \prime}(t)=\frac{3}{4} u(t)+u\left(\frac{t}{2}\right)+\left(-t^{2}+2\right), 0 \leq t \leq 1, \\
u(0)=0, u^{\prime}(0)=0,
\end{gathered}
$$

where the exact solution of above equation is $u(t)=t^{2}$ (see [28]). With the presented method, the graph of error is plotted in Fig. 2. 


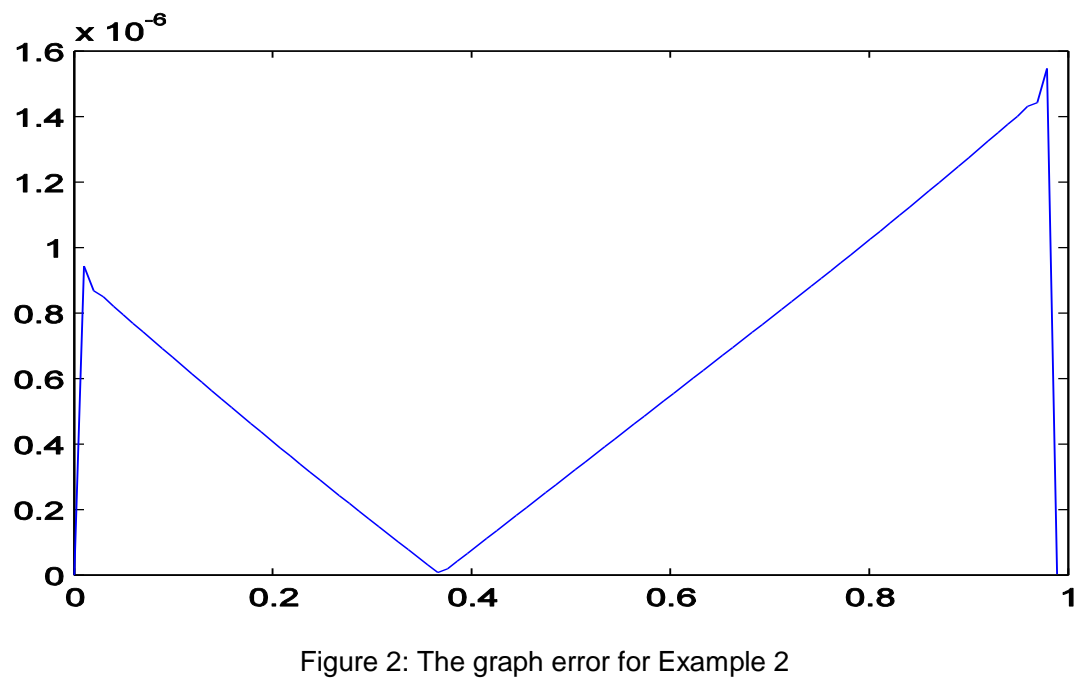

Example 3. Consider the following second order linear DDE

$$
\begin{gathered}
u^{\prime \prime \prime}(t)=u(t)+u^{\prime}\left(\frac{t}{2}\right)+u^{\prime \prime}\left(\frac{t}{2}\right)+\frac{1}{2} u^{\prime \prime \prime}\left(\frac{t}{4}\right) \\
-t^{4}-\frac{t^{3}}{2}-\frac{4}{3} t^{2}+21 t, 0 \leq t \leq 1, \\
u(0)=u^{\prime}(0)=u^{\prime \prime}(0)=0,
\end{gathered}
$$

where the exact solution of above equation is $u(t)=t^{4}$ (see [28]). With the presented method, the graph of error is plotted in Fig. 3.

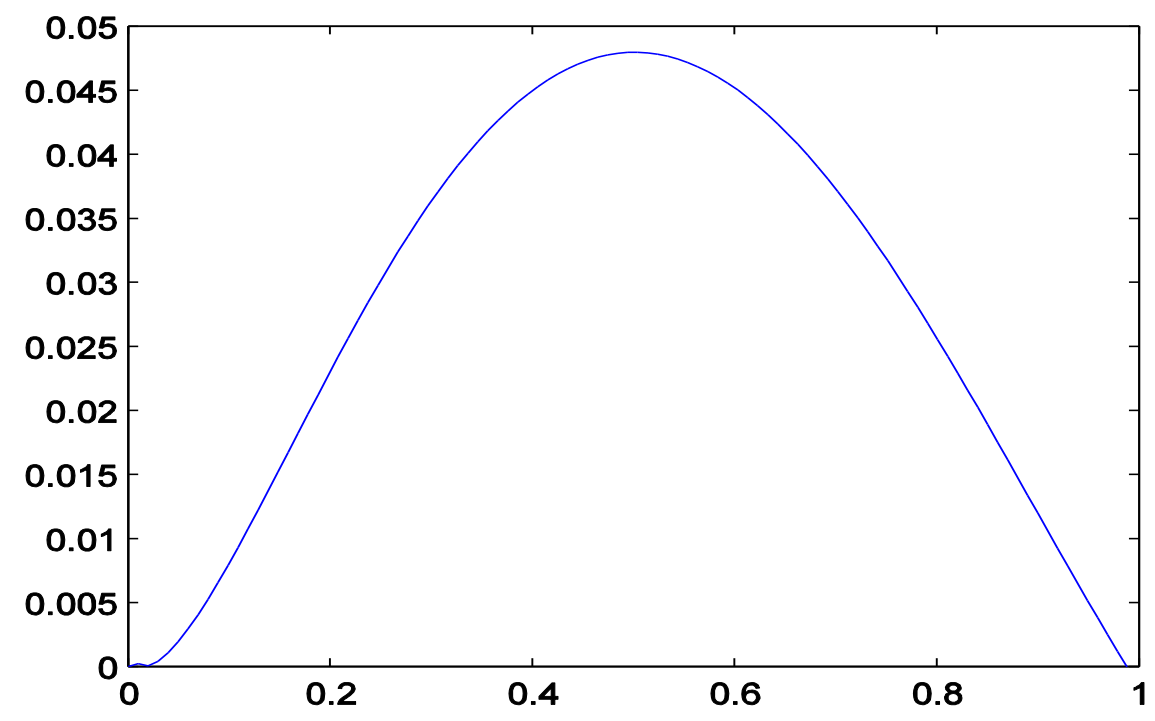

Figure 3: The graph error for Example 3

\section{Conclusions}


In this paper, we developed an efficient and computationally attractive method to solve delay differential equations. The method is based on the use of the radial basis functions. Some examples are given to verify the reliability and efficiency of the proposed method.

\section{References}

[1] J. V. Beltran and J. Monterde, "Bezier solutions of the wave equation," Lecture notes in Computational Sciences, Computational Science and its Applications-ICCSA (A. Laganà, M. L. Gavrilova, V. Kumar, Y. Mun, C. J. K.Tan \& O. Gervasi eds). Lecture Notes in Computer Science, vol. 3044. Berlin: Springer, pp. 631-640, 2004.

[2] A. Bemporad, A. Giua, and C. Seatzu, "Synthesis of state-feedback optimal controllers for continuous-time switched linear systems," In Proc. 41st IEEE conference on decision and control, Las Vegas, Nevada USA, 2002.

[3] R. Cholewa, A. J. Nowak, R. A. Bialecki, and L. C. Wrobel, "Cubic Bezier splines for BEM heat transfer analysis of the 2-D continuous casting problems," Computational Mechanics, vol. 28, pp. 282-290, 2002.

[4] C. H. Chu, C. C. L. Wang, and C. R. Tsai, "Computer aided geometric design of strip using developable Bezier patches," Computers in Industry, vol. 59, no. 6, pp. 601-611, 2008.

[5] M. Egerstedt, Y. Wardi, and F. Delmotte, "Optimal control of switching times in switched dynamical systems," In Proc. IEEE conference on decision and control, vol. 3, pp. 2138-2143, 2003.

[6] M. Evrenosoglu and S. Somali, "Least squares methods for solving singularity perturbed two-points boundary value problems using Bezier control point," Applied Mathematics Letters, vol. 21, no. 10, pp. 1029-1032, 2008.

[7] G. E. Farin, "Curve and surfaces for computer aided geometric design,” First ed, New York: Academic Press, 1988.

[8] B. Farhadinia, K. L. Teo, and R. C. Loxton, "A computational method for a class of non-standard time optimal control problems involving multiple time horizons," Mathematical Computer Modelling, vol. 49, pp. 1682-1691, 2009.

[9] M. Gachpazan, "Solving of time varying quadratic optimal control problems by using Bezier control points," Computational and Applied Mathematics, vol. 30, no. 2, pp. 367-379, 2011.

[10] J. Geromela, P. Colaneri, and P, Bolzern, "Passivity of switched linear systems: Analysis and control design," Systems and Control Letters, vol. 61, pp. 549-554, 2012.

[11] F. Ghomanjani, M. H. Farahi, " The Bezier control points method for solving delay differential equation," Intelligent Control and Automation, vol. 3, no. 2, pp. 188-196, 2012.

[12] F. Ghomanjani, M. H. Farahi and M. Gachpazan, "Bezier control points method to solve constrained quadratic optimal control of time varying linear systems," Computational and Applied Mathematics, vol. 31, no. 3, pp. 1-24, 2012.

[13] A. Giua, C. Seatzu, and C. Van Der Me, "Optimal conrol of switched autonomous linear systems," In Proc. IEEE conference on decision and control, pp. 2472-2477, 2001.

[14] K. Harada and E. Nakamae, "Application of the Bezier curve to data interpolation, Computer-Aided Design," International Journal of Computer Mathematics, vol. 14, no. 1, pp. 55-59, 1982.

[15] M. Heinkenschloss, "A time-domain decomposition iterative method for the solution of distributed linear quadratic optimal control problems," Applied Mathematics and Computation, vol. 173, pp. 169-198, 2005.

[16] I. Hwang, J. Li, and D. Du, "A numerical algorithm for optimal control of a class of hybrid systems: differential transformation based approach," International Journal of Control, vol. 81, no. 2, pp. 277-293, 2008.

[17] H. Juddu, "Spectral method for constrained linear-quadratic optimal control," Mathematics Computers In simulation, vol. 58, pp. 159-169, 2002.

[18] B. Lang, "The synthesis of wave forms using Bezier curves with control point modulation," In: The Second CEMS Research Student Conference, 1st edn. Morgan kaufamann, San Francisco, 2004.

[19] A. T. Layton and M. Van de Panne, "A numerically evident and stable algorithm for animating water waves," The visual Computer, vol. 18, pp. 41-53, 2002.

[20] M. Kamgarpour, and C. Tomlin, "On optimal control of non-autonomous switched systems with a fixed mode sequence," Automatica, Doi:10.1016/j.automatica, 2012.03.019, 2012. 
[21] M. Margaliot, "Stability analysis of switched systems using variational principles: An introduction," Automatica, vol. 42, pp. 2059-2077, 2006.

[22] B. Niua, and J. Zhaoa, "Stabilization and $L_{2}$-gain analysis for a class of cascade switched nonlinear systems: An average dwell-time method," Nonlinear Analysis: Hybrid Systems, vol. 5, pp. 671-680, 2001.

[23] G. N $\ddot{u}$ rnberger and F. Zeilfelder, "Developments in bivariate spline interpolation, Journal of Computational and Applied Mathematics," Computers and Mathematics with Applications, vol. 121, no. 1-2, pp. 125-152, 2000.

[24] H. Prautzsch, W. Boehm, M. Paluszny, Bezier and B-Spline Techniques,” Springer. 2001.

[25] Y. Q. Shi, H. Sun, "Image and video compression for multimedia engineering," CRC Press LLc, 2000.

[26] R. Winkel, "Generalized Bernstein Polynomials and Bezier Curves: An Application of Umbral Calculus to Computer Aided Geometric Design," Advances in Applied Mathematics, vol. 27, no. 1, pp. 51-81, 2001.

[27] C. Wu, K. L. Teo, R. Li, and Y. Zhao, “Optimal control of switched systems with time delay,” Appl, Math. Letters, vol. 19, pp. 1062-1067, 2006.

[28] X. Xu, G. Zhai, and S. He, "Some results on practical stabilizability of discrete-time switched affine systems," Nonlinear Analysis: Hybrid Systems, vol. 4, pp. 113-121, 2010.

[29] X. Xu, and P. J. Antsakalis, "Optimal control of switched systems based on parameterization of the switching instants," IEEE Transactions on Automatic Control, vol. 49, no. 1, pp. 2-16, 2004.

[30] J. Zheng, T. W. Sederberg, and R. W. Johnson, "Least squares methods for solving differential equations using Bezier control points,", Applied Numerical Mathematics, vol. 48, pp. 237-252, 2004. 